\title{
Pediatric stroke: current diagnostic and management challenges
}

\author{
Nikil K. Rajani ${ }^{1}$, Kirsten Pearce ${ }^{2}$, Tom Campion ${ }^{2}$, Vincenzo Salpietro ${ }^{3}$, Mariana Planells ${ }^{4}$, \\ Winston Chong ${ }^{5}$, Tufail Patankar ${ }^{6}$, Kshitij Mankad $^{2}$ \\ ${ }^{1}$ Department of Radiology, Imperial Healthcare NHS Trust, London, UK; ${ }^{2}$ Department of Radiology, Great Ormond Street Hospital, London, UK; \\ ${ }^{3}$ Research Unit, UCL Institute of Neurology, London, UK; ${ }^{4}$ Department of Radiology, Hospital General Universitario de Alicante, Alicante, Spain; \\ ${ }^{5}$ Monash Imaging, Monash University, Melbourne, Australia; ${ }^{6}$ Department of Neuroradiology, Leeds Teaching Hospitals NHS Trust, Leeds, UK
}

Correspondence to: Kshitij Mankad. Department of Radiology, Great Ormond Street Hospital, London, UK. Email: kshitij.mankad@gosh.nhs.uk.

Submitted Nov 10, 2018. Accepted for publication Nov 21, 2018.

doi: 10.21037/qims.2018.11.09

View this article at: http://dx.doi.org/10.21037/qims.2018.11.09

\section{Introduction}

Stroke is not a diagnosis which springs to mind within the pediatric age group. Nonetheless, the combined incidence of ischemic and hemorrhagic stroke is estimated at 1.3 to 13.0 children per 100,000 (1) and the overall incidence is increasing (2) due to a combination of improved survival of those with risk factors and increased recognition.

Pediatric stroke is a heterogeneous entity, both in terms of etiology and presentation. This makes accurate and timely diagnosis challenging, and leaves uncertainty regarding optimum acute management. The 2017 Royal College of Pediatrics and Child Health guidelines on pediatric stroke (3) aimed to ameliorate these issues and provide a model for standardized pediatric stroke management in the United Kingdom. This long-awaited document was the combined work of multiple organizations, reflecting the multidisciplinary approach required for pediatric stroke management.

Adult stroke management is a standard against which pediatric stroke management can be compared and provides a template for potential improvement. Within the adult population, stroke diagnosis and management is streamlined and algorithm-based and has a robust evidence base (4). Rapid identification facilitates the provision of potentially life-saving treatments such as fibrinolysis and mechanical thrombectomy. However, unlike children, adult stroke care is underpinned by the relative uniformity of clinical presentation and underlying pathology. In children, the heterogeneous etiology, combined with a variable presentation creates diagnostic delays and management challenges (3). These challenges need addressing in order to avoid the significant morbidity and mortality and the potential for lifelong disability which is associated with childhood stroke (5).

This article will summarize current challenges in pediatric stroke management and address the ways in which radiology can support timely diagnosis and assist patient selection for potentially life-saving treatments.

\section{Childhood stroke has a variable clinical presentation and heterogeneous etiology}

\section{Clinical presentation}

Whilst stroke classically presents with an acute focal neurological deficit such as hemiplegia, speech or gait disturbance (6), the presentation is highly dependent on the age of the child and may not be specific. Toddlers and infants tend to present most non-specifically, with features such as irritability, somnolence, lethargy, feeding difficulty, apneic spells and hypotonia (7). Approximately 30-50\% of children with an acute neurological presentation will have non-vascular diagnoses (8). The aim of clinical and imaging triage is to differentiate ischemic, hemorrhagic and non-vascular etiologies, triggering different management cascades. The RCPCH guidelines recognize the potential for non-specific presentations and urge the assessing clinician to consider stroke in unusual presentations, or when there are unexplained parental concerns (3). In addition, stroke mimics further confuse the picture. 
Table 1 Risk factors for stroke in children

Ischemic stroke [RCPCH guidelines (3) and Mallick et al. 2014 (6)]

Arteriopathies including focal cerebral arteriopathy, arterial dissection, vasculitidies and Moyamoya;

Cardiac disease including cardiac interventions/surgery, congenital conditions, Extracorporeal Membrane Oxygenation (ECMO), right to left shunts, arrhythmia;

Prothrombotic disorders such as anticardiolipin antibody;

Sickle cell disease;

Infection/sepsis;

Thrombophilias such as factor V Leiden deficiency and antiphospholipid syndrome;

Genetic and racial predispositions such as blacks and Asians and the male gender

Hemorrhagic stroke [RCPCH guidelines (3)]

Vascular disorders including arteriovenous malformation, aneurysms, Moyamoya and cavernomas;

Clotting disorders such as platelet disorders, inherited bleeding diatheses and anticoagulant therapy;

Sickle cell disease;

Illicit drugs such as cocaine and amphetamine abuse;

Black ethnicity and particularly male sex

\section{Etiologies (Table 1)}

The pathogenesis of both ischemic and hemorrhagic stroke differ considerably in children compared to adults (9). In adults, ischemic stroke typically results from a ruptured atherosclerotic plaque or via thromboembolism. In contrast, the majority of ischemic strokes in children occur in those with predisposing cardiac, vascular or hematological conditions (6). According to a prospective population-based study by Mallick et al., the most common risk factor for ischemic insult in children was acute systemic disorders, i.e., sepsis, dehydration, hypoxia or acidosis, which accounted for $31 \%$ of the cases examined. In order of decreasing frequency, the next most common were arteriopathy, chronic systemic disease (e.g., sickle cell) and cardiac causes which accounted for $29 \%, 25 \%$ and $23 \%$ of the cases respectively (6).

Cerebral arteriopathy increases the risk of both ischemic and hemorrhagic stroke, occurring in approximately $50 \%$ of cases of childhood acute ischemic stroke (10). It is characterized by narrowing or occlusion of the intracranial arterial vessels. Cerebral arteriopathy is an umbrella term used to describe a broad range of causes including dissection, vasculitis, Moyamoya, varicella vasculitis, radiation therapy and syndromic conditions (e.g., neurofibromatosis type-1 and trisomy 21) (10). The most common arteriopathy form in children is known as focal cerebral arteriopathy of childhood (FCA), and there is some evidence that FCA may have an inflammatory or post-viral etiology (11).

Ischemic and hemorrhagic stroke are important causes of morbidity and mortality in Sickle Cell Disease (SCD) with an estimated prevalence of $4.0 \%$ in childhood (12). The risk of ischemic stroke is principally due to SCD-associated cerebral arteriopathy, and Moyamoya syndrome may also be seen in SCD (13). Stroke in SCD is managed differently compared to other children, with blood transfusion often used in the acute setting, whilst the use of anti-platelets such as aspirin is not recommended (14).

Congenital heart disease (CHD) is a strong risk factor for stroke, with a 19-fold increase in risk of ischemic or hemorrhagic stroke in children (15). As well as the underlying condition, stroke risk is increased through arrhythmia, anticoagulation, cardiac catheterization, ventricular assist devices and corrective surgery (6). Here the mechanism is assumed to be embolic, which more readily reflects the underlying pathophysiology within the adult population (16) and the potential for outcomealtering and time-critical IV or endovascular therapy in this subgroup is therefore of great interest.

Prothrombotic disorders such as Antithrombin, protein $\mathrm{C}$, and protein $\mathrm{S}$ deficiency, factor $\mathrm{V}$ Leiden and elevated 
lipoprotein A are another important cause of ischemic stroke in children (6).

Coagulopathy and vascular malformations predispose to hemorrhagic stroke. Vascular anomalies account for $40-90 \%$ of childhood hemorrhagic stroke (17) (Figure 1).

\section{Delays in diagnosis}

The non-specific clinical presentation, variable risk factors and pathogenesis combine to create diagnostic uncertainty and management dilemmas. As a consequence, stroke is under-recognized in children and there can be marked delays in diagnosis (18). One study showed a median interval from symptom onset to diagnosis of 23 hours (18). Delays can result in parental anxiety, as well as denying potentially life-saving, time-critical treatments such as intravenous thrombolysis or mechanical thrombectomy. They can occur at several steps in the clinical pathway from the recognition of symptoms, clinical diagnosis, or obtaining diagnostic neuroimaging.

\section{How can these challenges be overcome?}

To combat difficulties in clinical diagnosis and symptom recognition, the 2017 guidelines suggest increasing awareness of stroke in childhood amongst the general public and front-line clinical staff, through emphasizing use of the FAST (face, arm, speech, time) tool in the pediatric population (3). Popularizing such tools serves as a reminder that stroke must be considered and emphasizes the time critical nature of stroke management. This facilitates early recognition and appropriate specialist management. In the context of children with known risk factors (e.g., SCD or congenital heart disease), the guidelines suggest implementation of medical alert bracelets, prompting the assessing clinician to consider stroke as the cause of a nonspecific presentation (3). These recommendations are promising, but may not succeed in emulating the cultural shifts seen within adult stroke management over the preceding decades.

\section{How can imaging address existing challenges?}

Diagnostic imaging in acute stroke is obtained primarily through computed tomography (CT) and magnetic resonance (MR) imaging. Assessment of the vasculature can be additionally undertaken using CT with contrast (CTA), or MR angiography (MRA), performed with or without contrast. Furthermore there are options for advanced techniques such as CT and MR perfusion imaging. Considerations in selecting the appropriate modality include diagnostic accuracy, availability and tolerability for the child. The 2017 UK guidelines stress the need for rapid diagnosis and pragmatism with CT as the initial investigation of choice for suspected stroke. They recommended CT and CTA within 1 hour of arrival at hospital, based on the widespread availability of CT, rapid acquisition, and high sensitivity for hemorrhage (3). This pathway is recommended in patients presenting with acute focal neurological deficit, aphasia or reduced conscious level. The guidelines also suggest consideration of emergent brain imaging in children with new onset focal seizures, severe headache or neck pain, transient loss of consciousness, altered behavior, ataxia, vertigo, or witnessed transient focal neurological deficit (3).

There are however inherent issues with such a guideline in children. In many cases of acute ischemic stroke, the initial unenhanced CT is normal (19). The rationale for initiating thrombolysis in the adult population is therefore based on the absence of hemorrhage on imaging with a strong clinical suspicion of ischemic insult (e.g., focal neurological deficit in an adult) (19). In children however, the clinical picture is more ambiguous. There is an understandable reluctance amongst many pediatricians to commence potentially hazardous therapy on the basis of a normal CT study, which has a low sensitivity for ischemic stroke. Due to the non-specific clinical presentation, there is a greater risk of thrombolysing patients with stroke mimics in the pediatric than adult populations. The potential for adverse iatrogenic effects means that, at present, a normal CT scan simply does not have the same implications for a stroke physician and pediatrician and may delay diagnosis.

A CTA is suggested as the next line of investigation because of rapid acquisition, widespread availability and the possibility of performing it shortly after the initial unenhanced CT. CTA can identify features associated with ischemic stroke such as major vessel occlusion, arteriopathy, or arterial dissection (19).

However, again, the absence of a vascular occlusion is non-specific for non-embolic infarcts since small caliber vessel thromboembolic occlusion can occur without imaging evidence on CTA. In the event of a non-embolic cause, the CTA will also be non-contributory and the use of fibrinolysis therapy is unlikely to be efficacious. In addition, CTA in pediatric patients can be challenging to interpret, especially if no dedicated neuroradiologist is routinely 

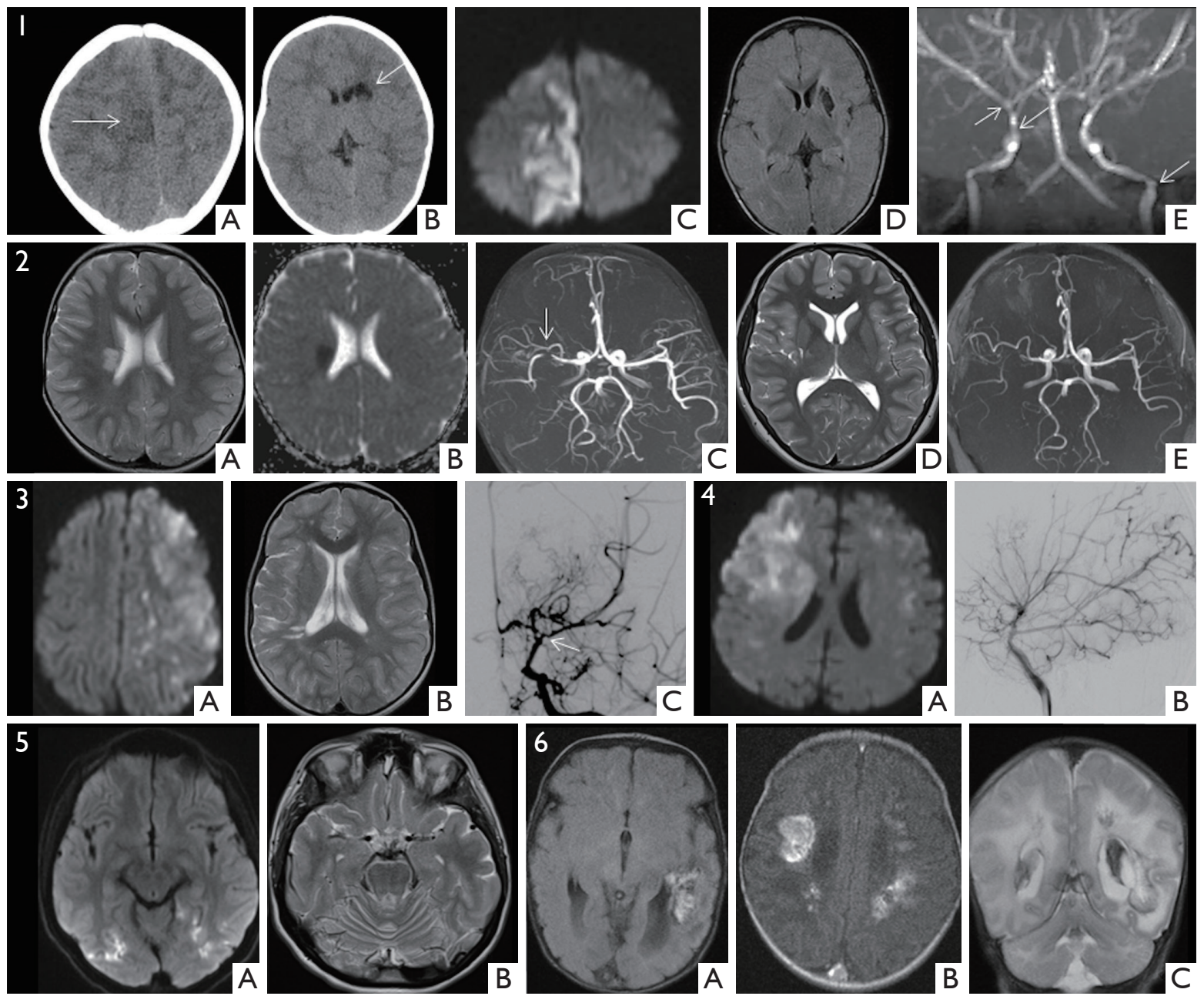

Figure 1 Pediatric stroke has diverse etiopathogenesis. One size does not fit all! Case 1: Fibromuscular dysplasia. A 14-month-old boy presenting with 3 episodes of acute focal seizures of his left face, arm and leg without loss of consciousness and with residual left hemiplegia. Non-contrast CT showed low attenuation lesion in the right parasagittal cortex consistent with an acute infarct (arrow) (1A). It also showed a chronic cavitated infarct in the left caudate nucleus (arrow) (1B). Diffusion weighted imaging (1C) confirmed right anterior cerebral artery acute infarct; FLAIR imaging confirmed chronic left basal ganglia infarct (1D); MRA showed beading appearance of vessels (arrows) (1E). Case 2: Focal cerebral arteriopathy (FCA). A 5-year-old girl with acute onset of left sided weakness following chicken pox infection 5 weeks previously. T2 weighted (2A) and ADC (2B) revealed acute infarct in the right corona radiata with focal stenosis of the right MCA on the MRA (arrow) (2C). Follow up imaging 10 years later (2D and 2E) shows a mature focal infarct but stable angiographic findings. Case 3: Moyamoya disease. Arterial ischemic strokes of variable ages in a 7-year-old girl with sickle cell disease shown on the DWI and T2 weighted images (3A,3B). Digital Subtraction Angiogram (DSA) (3C) confirms occlusive arteriopathy of the internal carotid T-junction (arrow) with extensive typically described 'puff of smoke' basal collateralization. Case 4: ACTA 2 mutation. A 6-year-old boy showing the typical imaging phenotype of ACTA 2 related stroke, a mixture of acute and chronic infarcts (4A), proximal dilatation of the large arteries (4B) with terminal tapering and smooth peripheral arteries (branches of a tree appearance). Note the absence of basal collaterals. ACTA 2 is a mutation affecting the smooth muscle alpha-actin complex. Case 5: Mitochondrial encephalopathy with lactic acidosis and stroke like episodes (MELAS). A 16-year-old girl with acute infarcts in multiple areas of the brain shown on DWI $(5 \mathrm{~A})$ not confirming to vascular territories, and cerebellar atrophy (5B), consistent with a mitochondrial stroke pattern. The MR angiogram was normal. Case 6: Hemorrhagic venous infarction. A 25-day-old term neonate presenting with seizures. Extensive venous hemorrhagic infarction is noted in both cerebral hemispheres, particularly involving the trans-medullary veins $(6 \mathrm{~A}, 6 \mathrm{~B}, 6 \mathrm{C})$. The CSF tested positive for parechovirus infection. Sepsis is a common cause of infarction in the very young children. 
available to report these studies.

For the reasons outlined above, there is an argument that pediatric patients may benefit from using MR as the initial investigative imaging modality, which is both more sensitive and specific for detecting ischemic stroke. In particular, diffusion weighted sequences can detect the cytotoxic edema associated with acute ischemia, with high sensitivity (19), providing a greater diagnostic yield and preventing false reassurance. In children, this has been demonstrated with observational studies (6). Further MR imaging sequences provide greater diagnostic detail which can help differentiate infarct from stroke mimics such as tumors (19). This approach has been recommended by the recent Australian and North American pediatric stroke guidelines, which highlight MR as the initial diagnostic imaging modality of choice, using an abbreviated protocol to counter the long acquisition time when the child presents within the timeframe for reperfusion therapy (20,21). A minimum suggested protocol would include diffusion weighted sequence, susceptibility-weighted imaging, FLAIR and a time-of-flight MRA (21).

There are clearly problems with access to MR, with frequent need for anesthetic support in children and problems with out-of-hours scanning. The RCPCH guidelines currently recommend that MR should only be offered as the initial imaging modality if available within one hour of arrival in hospital (3). However, tools developed for assessing parenchymal change on CT cannot be automatically assumed to be transferable to MR. Defining a 'safe' volume of diffusion change for thrombolysis is a parameter which has yet to be evidenced. Finally, a key advantage of CT is its sensitivity to acute hemorrhage; whilst there is evidence that MR may be equivalent in sensitivity (22), and this is supported by the Australian and American guidelines, this has not been conclusively proven and acute plain CT may remain necessary before reperfusion therapy.

\section{Thrombolysis and thrombectomy in pediatric acute ischemic stroke}

The Thrombolysis in Pediatric Stroke study (23) collapsed due to inadequate recruitment and subsequent funding withdrawal. This was despite having a robust multicenter design. The key difficulty was the relative infrequency of children with acute ischemic stroke who qualified for thrombolysis and presented within the recommended 4.5-hour therapeutic window. This will likely prevent further prospective trials from being undertaken, thus increasing reliance on the existing retrospective evidence from cross-sectional studies and case series.

With regard to thrombectomy, evidence is limited to series of consecutive cases from individual centers. Retrospective evidence is fraught with confound and potential bias, an uneasy evidence base upon which to justify potentially hazardous acute treatment options. The authors of the 2017 guidelines acknowledge this, using a surveybased Delphi method to arrive at an expert consensus in order to provide guidance in consideration of thrombolysis and thrombectomy (3).

The guidelines state that intravenous thrombolysis can be considered on a case-by-case basis, within 4.5 hours of symptom onset and in the absence of any contraindications. Suggested inclusion criteria are: age 2 years or more, an acute focal neurological deficit and PedNIHSS score between 4 and 24. Imaging must confirm the absence of hemorrhage and demonstrate normal or minimally ischemic parenchyma on CT or MR evidence of acute ischemia. The efficacy of this guidance is muted somewhat by the age restrictions since prior studies have indicated that a greater proportion of pediatric strokes occur in the younger age groups, particularly in neonates (6). Interestingly, the guidelines stipulate that there must be arterial occlusion or severe stenosis on CTA or MRA (3). Arterial occlusion or stenosis is often absent in patients with confirmed ischemic stroke (24). Importantly, arterial occlusion is associated with worse outcomes following intravenous thrombolysis, and in adult stroke care the presence of a large vessel arterial occlusion favors the use of mechanical thrombectomy to improve the eventual outcome (25). Further, arterial stenosis in children may only represent a post viral FCA as described above.

Making arterial occlusion a mandatory criterion for the use of thrombolysis in children means that children lacking this feature will be denied potentially life-changing therapy. Furthermore, this results in selection of a treatment group where outcomes are known to be worse post-thrombolysis (Figure 2).

There is insufficient evidence for firm recommendations on mechanical thrombectomy in pediatrics worldwide but the guidelines draw upon the excellent outcomes in adult studies for added mechanical thrombectomy compared to intravenous thrombolysis alone. It has been suggested by the UK guidelines that thrombectomy can be considered in children with acute disabling ischemic stroke (PedNIHSS $\geq 6$ ) up to 12 hours after symptom-onset, 

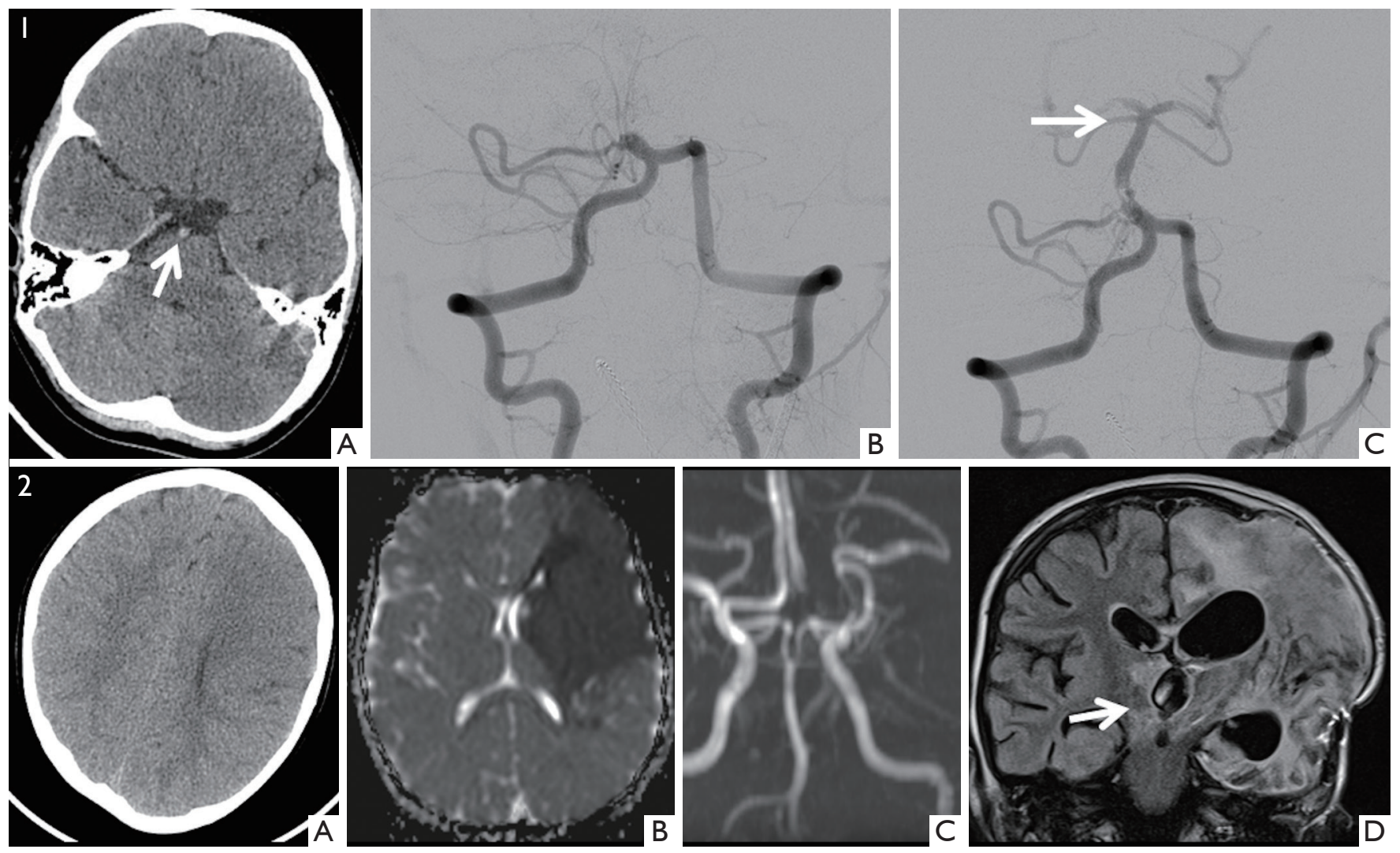

Figure 2 Acute management of stroke in children needs to be evaluated on a case by case basis. Case 1: Mechanical thrombectomy. A 5 -year-old boy suffering from a diarrhoeal illness presented with acute loss of consciousness. He was intubated and an unenhanced CT scan was performed, showing a dense basilar artery (arrow) (1A). The patient was transferred to the regional stroke centre within an hour. DSA performed 3 hours after onset of symptoms confirmed occlusion of the basilar artery (1B). The clot was mechanically retrieved with a $4 \times 20 \mathrm{~mm}^{2}$ Solitaire stent. Post intervention angiogram revealed recanalization (arrow) (1C). There was some hind brain swelling that needed surgical posterior fossa decompression, but no neurological deficits followed. Case 2: Large infarction needing decompression. This 5-yearold boy had a large left MCA territory infarction. Initial CT scan (2A) was performed 3 hours after onset of signs. Diffusion MR (2B) was performed 3 further hours later confirming the large infarct and lack of signal in the left MCA due to a proximal occlusion (2C). Salvageable brain tissue could not be confirmed. The patient went on to develop brain swelling needing decompression craniectomy on day 5 (2D), by which time the contralateral posterior circulation was already infarcted (arrow). Brain swelling increases from day 2 onwards and often the contralateral posterior cerebral artery is secondarily compressed against the tent as the hemisphere swells in such cases.

unless contraindicated, when there is a favourable profile of salvageable brain tissue on imaging (3). How this latter aspect of 'salvageable tissue' as assessed in the acute setting remains unclear.

\section{Conclusions}

The 2017 UK guidelines give a welcome sense of urgency and importance of the need for a more cohesive approach to the management of childhood stroke. The overarching aim for the pediatric stroke community must be to translate the improvements seen in adult stroke care over the last decade into the childhood population. Given the relative infrequency and heterogeneity of childhood stroke, a firm evidence base is unlikely to be forthcoming in the near future. Therefore, case-by-case consideration is the current modus operandi, and careful case selection will be crucial for potentially harmful acute treatments such as thrombolysis or thrombectomy. Although the 2017 RCPCH guidelines have helped raised the profile of pediatric stroke among clinicians, some suggestions, such as the need for arterial occlusion on imaging, may in fact preclude treatment of 
some children who would otherwise have benefitted from IV thrombolysis. We would therefore argue against the necessity of arterial occlusion on imaging to guide the need for thrombolysis. Triage should be based upon symptoms, likely cause, clinical severity, and the absence of clinical or radiological contraindications.

Given the challenges posed by pediatric stroke, we feel a cultural change towards more hyperacute MR may be warranted, whenever available, to assist in the diagnostic work up of this patient cohort. In doing so we hope to empower the assessing pediatrician to make confident decisions and ultimately lead to better outcomes for pediatric stroke patients.

\section{Acknowledgements}

None.

\section{Footnote}

Conflicts of Interest: The authors have no conflicts of interest to declare.

\section{References}

1. Mallick AA, O'Callaghan FJ. The epidemiology of childhood stroke. Eur J Paediatr Neurol 2010;14:197-205.

2. Gandhi SK, McKinney JS, Sedjro JE, Cosgrove NM, Cabrera J, Kostis JB. Temporal trends in incidence and long-term case fatality of stroke among children from 1994 to 2007. Neurology 2012;78:1923-9.

3. RCPCH. Stroke in childhood - clinical guideline for diagnosis, management and rehabilitation I RCPCH. Royal College of Paediatrics and Child Health; 2017.

4. Wardlaw JM, Murray V, Berge E, del Zoppo GJ.

Thrombolysis for acute ischaemic stroke. Cochrane Database Syst Rev 2014;(7):Cd000213.

5. Greenham M, Gordon A, Anderson V, Mackay MT. Outcome in Childhood Stroke. Stroke 2016;47:1159-64.

6. Mallick AA, Ganesan V, Kirkham FJ, Fallon P, Hedderly T, McShane T, Parker AP, Wassmer E, Wraige E, Amin S, Edwards HB, Tilling K, O'Callaghan FJ. Childhood arterial ischaemic stroke incidence, presenting features, and risk factors: a prospective population-based study. Lancet Neurol 2014;13:35-43.

7. Tsze DS, Valente JH. Pediatric stroke: a review. Emerg Med Int 2011;2011:734506.

8. Mackay MT, Chua ZK, Lee M, Yock-Corrales A, Churilov
L, Monagle P, Donnan GA, Babl FE. Stroke and nonstroke brain attacks in children. Neurology 2014;82:1434-40.

9. Paulson OB. Cerebral apoplexy (stroke): pathogenesis, pathophysiology and therapy as illustrated by regional blood flow measurements in the brain. Stroke 1971;2:327-60.

10. Amlie-Lefond C, Bernard TJ, Sebire G, Friedman NR, Heyer GL, Lerner NB, DeVeber G, Fullerton HJ.

Predictors of cerebral arteriopathy in children with arterial ischemic stroke: results of the International Pediatric Stroke Study. Circulation 2009;119:1417-23.

11. Gumer LB, Del Vecchio M, Aronoff S. Strokes in children: a systematic review. Pediatr Emerg Care 2014;30:660-4.

12. Ohene-Frempong K, Weiner SJ, Sleeper LA, Miller ST, Embury S, Moohr JW, Wethers DL, Pegelow CH, Gill FM. Cerebrovascular accidents in sickle cell disease: rates and risk factors. Blood 1998;91:288-94.

13. Dobson SR, Holden KR, Nietert PJ, Cure JK, Laver JH, Disco D, Abboud MR. Moyamoya syndrome in childhood sickle cell disease: a predictive factor for recurrent cerebrovascular events. Blood 2002;99:3144-50.

14. Kassim AA, Galadanci NA, Pruthi S, DeBaun MR. How I treat and manage strokes in sickle cell disease. Blood 2015;125:3401-10.

15. Fox CK, Sidney S, Fullerton HJ. Community-based casecontrol study of childhood stroke risk associated with congenital heart disease. Stroke 2015;46:336-40.

16. Dowling MM, Hynan LS, Lo W, Licht DJ, McClure C, Yager JY, Dlamini N, Kirkham FJ, Deveber G, Pavlakis S. International Paediatric Stroke Study: stroke associated with cardiac disorders. Int J Stroke 2013;8 Suppl A100:39-44.

17. Meyer-Heim AD, Boltshauser E. Spontaneous intracranial haemorrhage in children: aetiology, presentation and outcome. Brain Dev 2003;25:416-21.

18. Rafay MF, Pontigon AM, Chiang J, Adams M, Jarvis DA, Silver F, Macgregor D, Deveber GA. Delay to diagnosis in acute pediatric arterial ischemic stroke. Stroke 2009;40:58-64.

19. Smith AG, Rowland Hill C. Imaging assessment of acute ischaemic stroke: a review of radiological methods. $\mathrm{Br} \mathrm{J}$ Radiol 2018;91:20170573.

20. Rivkin MJ, Bernard TJ, Dowling MM, Amlie-Lefond C. Guidelines for Urgent Management of Stroke in Children. Pediatr Neurol 2016;56:8-17.

21. Australian Childhood Stroke Advisory Committee. The Diagnosis and Acute Management of Childhood Stroke, Clinical Guideline 2017.

22. Kidwell CS, Chalela JA, Saver JL, Starkman S, Hill MD, Demchuk AM, Butman JA, Patronas N, Alger JR, 
Latour LL, Luby ML, Baird AE, Leary MC, Tremwel M, Ovbiagele B, Fredieu A, Suzuki S, Villablanca JP, Davis S, Dunn B, Todd JW, Ezzeddine MA, Haymore J, Lynch JK, Davis L, Warach S. Comparison of MRI and CT for detection of acute intracerebral hemorrhage. Jama 2004;292:1823-30.

23. Rivkin MJ, deVeber G, Ichord RN, Kirton A, Chan AK, Hovinga CA, Gill JC, Szabo A, Hill MD, Scholz K, AmlieLefond C. Thrombolysis in pediatric stroke study. Stroke 2015;46:880-5.

24. Zaidat OO, Lazzaro M, McGinley E, Edgell RC, Nguyen T, Linfante I, Janjua N. Demand-supply of

Cite this article as: Rajani NK, Pearce K, Campion T, Salpietro V, Planells M, Chong W, Patankar T, Mankad K. Pediatric stroke: current diagnostic and management challenges. Quant Imaging Med Surg 2018;8(10):984-991. doi: 10.21037/qims.2018.11.09 neurointerventionalists for endovascular ischemic stroke therapy. Neurology 2012;79:S35-41.

25. Broderick JP, Palesch YY, Demchuk AM, Yeatts SD, Khatri P, Hill MD, Jauch EC, Jovin TG, Yan B, Silver FL, von Kummer R, Molina CA, Demaerschalk BM, Budzik R, Clark WM, Zaidat OO, Malisch TW, Goyal M, Schonewille WJ, Mazighi M, Engelter ST, Anderson C, Spilker J, Carrozzella J, Ryckborst KJ, Janis LS, Martin RH, Foster LD, Tomsick TA. Endovascular therapy after intravenous t-PA versus t-PA alone for stroke. $\mathrm{N}$ Engl J Med 2013;368:893-903. 